\title{
Fuzzy Linguistic Summaries in Rule-Based Adaptive Hypermedia Systems
}

\author{
Miguel-Ángel Sicilia ${ }^{1}$, Paloma Díaz $^{1}$, Ignacio Aedo ${ }^{1}$, Elena García $^{2}$ \\ 1 Laboratorio DEI, Computer Science Department, Universidad Carlos III de Madrid \\ Av. Universidad 30, 28911 Leganés (Madrid), Spain \\ \{msicilia, pdp\}@inf.uc3m.es, aedo@ia.uc3m.es \\ http://www.dei.inf.uc3m.es \\ 2 Computer Science Department, Universidad de Alcalá de Henares \\ Ctra. Barcelona km. 33.600, 28871 Alcalá de Henares (Madrid), Spain \\ elena.garciab@uah.es
}

\begin{abstract}
Rule-based adaptive hypermedia systems personalize the structure of the hypermedia space using an inference mechanism that operates on a specific knowledge representation about its users. Approximate quantifiers are very frequently used in human language expressions that entail the summarization of a large number of facts. We describe how quantified expressions can be used in adaptation rules to specify common adaptation behaviors, enhancing rule's expressive power for the human expert. Those quantified expressions can be implemented through fuzzy quantification mechanisms operating on fuzzy linguistic labels and relations, and can be integrated as extensions in generalpurpose rule-based adaptive hypermedia systems.
\end{abstract}

\section{Introduction}

Adaptive hypermedia systems (AHS) personalize the information, links and navigation features of the hypermedia space by using knowledge about its users, represented in a user model. Several adaptation techniques can be used for this goal, which are in turn abstracted in adaptation methods at a conceptual level (as defined in [2] and extended in [3]). In addition, a particular technique can adapt different aspects of the hypermedia structure (aspects that are called adaptation technologies). More specifically, rule-based AHS use an inference mechanism to implement adaptation and/or user modeling behaviors, resting on some kind of knowledge representation model. These systems can be considered as general-purpose ones when they are defined on a conceptual AH architecture as in [22]. In many cases, adaptation rules are defined by domain experts (e.g. marketing directors in Web recommendation systems or teachers in educational AHS), in a process of knowledge acquisition that results in a reusable and easily modifiable knowledge base about the intended adaptation behavior of the system. Indeed this approach is taken in several Web personalization engines [6]).

In this paper, we describe a general-purpose syntax and execution semantics for using fuzzy quantifiers in rule-based AHS. Although different theories of uncertainty representation have been applied in user modeling [11, 17], quantified expressions have 
not been applied as a general-purpose rule-modeling construct, even though their closeness to human language expression suggests that they could significantly enhance the rule definition process. The use of quantifiers in adaptation rules can be helpful for different adaptation technologies, and can be used also for the task of user modeling. In [19], an application of fuzzy linguistic quantified expressions is described for the specific user-modeling task of classifying users of a Web application in vague categories (fuzzy stereotypes), based on their navigation history. In this work, we focus on the description of adaptation behaviors using quantified expressions.

The rest of this paper is structured as follows. Section 2 describes fuzzy quantified expressions in rules and how they can be applied to extended-for-fuzziness AH models. Examples of adaptation methods that can be implemented using them are described in Section 3, along with the most relevant implementation details. Finally, conclusions and future research directions are provided in Section 4.

\section{Fuzzy Quantified Rules in Adaptive Hypermedia}

\subsection{Quantified Expressions and Rule Formulation}

Approximate quantifiers like 'almost all' or 'many' are very frequently used in human language, and serve the important purpose of abstracting from details, and summarizing a large number of evidences into a global view [12]. A quantified linguistic expression can be specified as an expression in the form " $Q X$ " in the case of absolute quantification (for example, 'quite a few visits') or in the form " $Q X$ are $Y$ " in the case of relative - or proportional - quantification (for example, "most visits are short'). Both $X$ and $Y$ are natural language nouns or phrases, which, for our purposes, must refer to user or domain model information that can be crisp or vague, and that must be interpreted in the context of a specific AH schema. Those expressions can be integrated in the antecedent of a rule, in the general form "if $Q X$ then $<<$ action $>>$ ", where action stands for the activation of a specific adaptation technology, or the addition of an inferred fact to the user model.

Fuzzy quantified expressions can be added to existing adaptation rule syntax to enrich the expressiveness of the rule language. An experiment was carried out to gather some evidence about the appropriateness of including quantifiers in the syntax of the adaptation rules. We focused on a specific and basic adaptation technology, namely a direct guidance, a kind of adaptive navigation support [2]. Both computer specialists and non-technical experts were included in the population (although experience in using the hypermedia technology we're analyzing - the Web - was considered mandatory). In consequence, we partitioned the sample according to two different user profiles: technicians and non-technicians. The objective of the study was to find how frequently natural language quantifiers were used in the formulation of simple adaptation rules. A scenario was developed in which the main page of a research group's Web site was described. The site had a navigation bar in a left frame that guided the 
user to different sections of the site. Participants were asked to write down 'if/then' rules in natural language to perform direct guidance to sections to which the user might be interested in, based exclusively in previous navigation history. Results showed that eighty three percent of the thirty participants used a quantifier (as 'most', 'many' and 'often') to describe the rule (either referring to frequency of visits, sessions or interactions in general), with no significant difference between the two user profiles. Although this is only a small experiment, it reveals the importance of quantifiers in human expression - a review of available evidence is in [16].

\subsection{Fuzzy Linguistic Quantifiers}

Zadeh's sigma-count operator [24] is the earliest operational definition of the concept of linguistic quantifier in terms of the theory of fuzzy sets and possibility theory. Although this and other fuzzy linguistic definitions of quantifiers do not achieve perfect linguistic adequacy (see, for example, [7]), a number of application exists that prove their usefulness in modeling natural language constructs. The approach presented here uses sigma-count and OWA operators, but other quantification mechanisms can be used either, with no impact in the conceptual semantics of the adaptation methods.

Most existing approaches define fuzzy linguistic quantifiers as fuzzy subsets of the non-negative real numbers (absolute quantifiers) or of the unit interval (proportional or relative quantifiers), and therefore can be considered as fuzzy numbers. In consequence, a quantifier $Q$ is represented either as a mapping $\mu_{Q}: R^{+} \rightarrow I$ or $\mu_{Q}: I \rightarrow I$ respectively, where $I$ is the unit interval. From an information modeling viewpoint, an essential difference exists between absolute and relative quantifiers, since the former are entity-dependent, that is, their definition is tied to the subject on which they're applied (we can consider that four are definitely many cars for a buyer but four is not many if we're expressing a football team's supporters number). In addition, it can be instance-dependant in the following sense: if we're measuring the user's visits in a Web site, many visits depend on the particular site we're considering. As a consequence, we can use the notation $\mu_{Q}^{x}$ to denote quantifier $Q$ applied to subject $x$.

Example definitions as $S$-functions of the absolute quantifier 'many' (applied to visits) and the relative one 'most' are provided in Figure 1, defined as Zadeh's $S$ functions $S(\mathrm{x} ; 10 ; 55 ; 100)$ and $S(\mathrm{x} ; 0.6 ; 0.75 ; 0.9)$ respectively.

Given a domain $D$ of elements (in our case, elements in the user or domain model, for example, the set of users or nodes in our AH system), a unary quantification mechanism in the form:

$$
M: X \in \wp(D) \rightarrow R^{+}
$$

where $\wp(D)$ is the fuzzy power set of $D$, provides a way of representing a summary of the cardinality of a fuzzy subset of elements in that domain through a real number. This numeric summary can then be matched to a quantifier $Q$ to obtain the degree of conformance of the quantified expression, thus achieving the transformation:

$$
Q: X \in \wp(D) \rightarrow I
$$


The definition can be extended to $n$-ary quantifiers, but we're only concerned with unary (absolute) and binary (relative) quantifiers. Zadeh's sigma-count is one of those quantification mechanisms, with the following absolute and relative formulations:

$$
\begin{gathered}
\text { sigma }- \text { count }(X)=\sum_{e \in D} \mu_{X}(e) \\
\text { sigma }- \text { count }\left(X_{2} / X_{1}\right)=\operatorname{sigma}-\operatorname{count}\left(X_{2} \cap X_{1}\right) / \operatorname{sigma}-\operatorname{count}\left(X_{1}\right)
\end{gathered}
$$

In the relative formulation, $X_{I}$ is the subset on which the proportion is computed. Additional details about the computation of linguistic summaries can be found, for example, in [18].
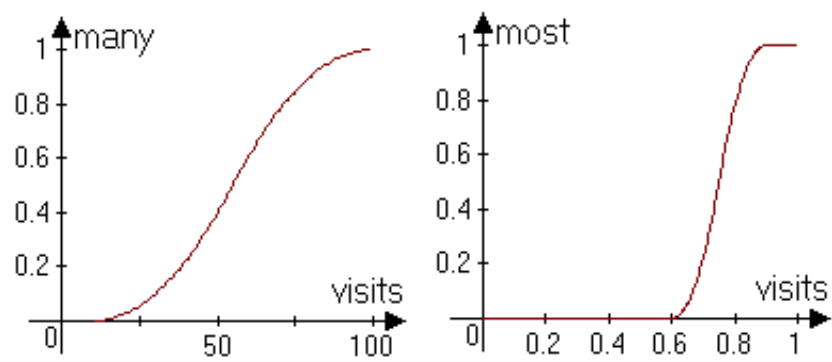

Figure 1. Example quantifiers: many and most

The other quantification mechanism we have used is based in Yager's proposal based on the Ordered Weighted Averaging (OWA) operator [23], only for proportional ones (we do not describe it here in detail, since it does not introduce changes in rule formulation).

\subsection{Fuzzy Quantified Rules in an AHS}

Adaptation techniques are characterized by a specific kind of knowledge representation and a specific adaptation algorithm. In this subsection, we describe a minimal abstract model that supports fuzzy linguistic labels and common fuzzy inference techniques, which can be implemented with a generalized fuzzy relation model framework (see, for example, [15]). This partial model could be combined with those from generic user models [13], or general purpose AHS like [22].

We'll assume a common division of our $\mathrm{AH}$ model in three components: the User Model (UM), the Domain Model (DM) and the Adaptation Model (AM). We also assume that an object model exists defining the entities in the three components in terms of classes, associations and other common object-oriented modeling constructs. The three model's components can be described with attributes in a relation as described in [22], but we describe here a more abstract rule syntax, that could easily be mapped onto these more specific schemas. In addition, some specific fuzzy types are introduced as extensions in the model, namely:

- Independent terms, for example, frequent, that is defined by an associated membership function $\mu_{\text {frequent }}$. Each term is defined on a domain that, in our 
case, could be groups of users or some entity belonging to the domain model.

- $\quad$ Linguistic labels picked from predefined label sets. For example (bargain, cheap, somewhat cheap, medium-priced, somewhat expensive, expensive, unaffordable) as price description. This second model construct allow us to store only references to the specific label in a label set.

Label sets fulfil some properties: (1) they must form totally ordered sets with odd cardinality $T_{g}+1$, in the form $L S_{g}=\left\{s_{i}\right\}, i \in\left\{0, \ldots, T_{g}\right\}$ such that the set is ordered: $s_{i}$ $>=s_{j}$ if $i>=j ;(2)$ it exists a negation operator: $\operatorname{Neg}\left(s_{i}\right)=s_{j}$, such that $j=T_{g}-i$, and (3) it exists maximization and minimization operators: $\operatorname{Max}\left(s_{i}, s_{j}\right)=s_{i}$ if $s_{i}>=s_{j}$, and $\operatorname{Min}\left(s_{i}, s_{j}\right)=s_{i}$ if $s_{i}<=s_{j}$. Label sets are specially well suited to model and aggregate user's preferences, since they've been extensively used in processes of group decision making (see for example [9]), and can be also used in content rating [5].

The set of users of our UM is denoted by $U=\left\{u_{i}\right\}$. Each user has a set of attributes each of them with an associated domain (including label sets), and can be included in one or more sets that represent associated information about the user. In the formulation of adaptive rules, with the general syntax $C \rightarrow A$, the set of antecedents $(C)$ express conditions on the user model and the context in which the adaptation is performed, and actions $(A)$ are updates on the UM (user modeling rules) or dynamic adaptation behaviors (adaptation rules). We describe here only the syntax of antecedents, in which the quantifiers are inserted. An antecedent can have the following formulations:

instance.attribute op expression

instance op expression

Where valid instances are the user currently connected $\left(u_{i}\right)$, the current navigation context $\left(c_{i}\right)$, an entity in the DM, or one of the groups defined by linguistic independent terms $g_{\text {term }}$. The context is a placeholder for navigation specific information, including session information and characteristics of the DM. Attributes are defined on entities on a domain, and operators ( $o p)$ and expressions are defined on that domains (e.g. character string, number comparison and the like). Association relationships between entities in the model are considered as a special kind of attribute, that denotes the collection of instances associated to the instance. An example of 'crisp' antecedent is $u_{i}$.age $>20$, and an example of a simple fuzzy expression is $u_{i}$ is loyal. We'll focus here only on fuzzy attributes and its applications and on fuzzy antecedents that are used in standard fuzzy rules like Mamdani min implication [21] (antecedents in the same rule are implicitly connected by and operators). Expressions regarding fuzziness include the following:

- Membership of the user in a group: $u_{i}$ includedIn $g_{t e r m}$, e.g. 'includedIn frequent' or 'includedIn advanced'.

- Fuzzy label sets domains, with two forms: $u_{i}$.att op label, e.g. 'likesLongNews is high', and $u_{i}$.rel $a_{j}$ op label, where rel is a (fuzzy) relationship between the user and some other entity, from which $a_{j}$ is an instance v.g. 'interestIn music is low'.

Quantification can operate on that expressions; Table 1 describes the main alternative syntaxes and examples (fuzzy subsets are in italics), which can be considered as ex- 
tensions of existing data design notations in hypermedia development methods [4] with imprecise (or vague) information (according to Smets [20]).

Table 1. Example of quantified expressions that can be used in rules

\begin{tabular}{|l|l|l|}
\hline Syntax & Example & Scenario \\
\hline $\mathbf{Q}$ gi & Many positive_answers & $\begin{array}{l}\text { Suppose the system is asking its users } \\
\text { for opinion about a new feature, and } \\
\text { positive answer is defined as a fuzzy set } \\
\text { on a ten-point scale valuation obtained } \\
\text { through a form. }\end{array}$ \\
\hline $\mathbf{Q}$ gi are gj & $\begin{array}{l}\text { Most frequent_users are } \\
\text { beginners. }\end{array}$ & Proportional quantification. \\
\hline $\begin{array}{l}\mathbf{Q} \text { instance.att instance.rel [in- } \\
\text { stance] }\end{array}$ & $\begin{array}{l}\text { Few [user's] visits } \\
\text { Few [user's] visits to } \\
\text { channel A. }\end{array}$ & $\begin{array}{l}\text { Queries about the absolute amount of } \\
\text { current user's visits, and the relative } \\
\text { amount of visits to an specific section. }\end{array}$ \\
\hline $\begin{array}{l}\mathbf{Q} \text { instance.att are gk } \\
\mathbf{Q} \text { gh instance.att are gk }\end{array}$ & $\begin{array}{l}\text { About half the [user's] } \\
\text { sessions are short. } \\
\text { Most excellent [user's] } \\
\text { assignment are short. }\end{array}$ & $\begin{array}{l}\text { Query about the typical session of the } \\
\text { current user, and query about the corre- } \\
\text { lation of assignment scoring and length } \\
\text { for a user. }\end{array}$ \\
\hline
\end{tabular}

The first and second syntaxes are related to subpopulations or groups of entities (characterize user's groups or global domain entities descriptions). The rest are about countable evidence related to a particular entity (i.e. a specific user or hypermedia node).

\section{Adaptation Methods and Techniques using Fuzzy Quantifiers}

A quantifier is specified as a predefined function and its parameters (if other functions need to be specified, MATHML ${ }^{1}$ content markup could be used instead). For example:

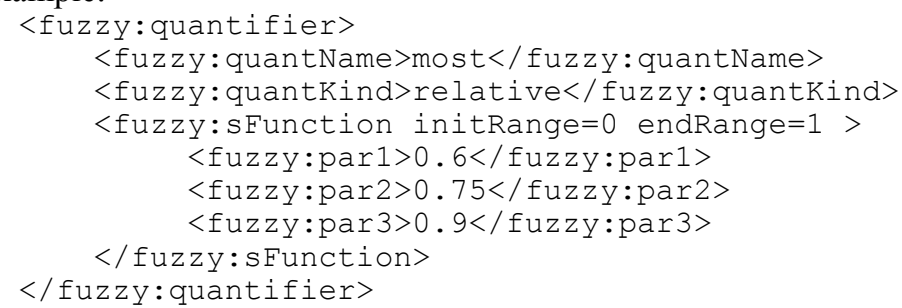

Linguistic labels can be defined in a similar way, but they need to be defined on attributes of 'the user' or an entity in the DM. For example, 'loyal' users can be defined by a left linear function on attribute numberofPurchasesYear as follows:

$<$ fuzzy: label>

<fuzzy: labelName>loyal</fuzzy: labelName>

<fuzzy:entity> user </fuzzy: entity $>$

<fuzzy:attribute>numberofPurchasesYear</fuzzy:attribute>

<fuzzy:definition>

$1<$ http: //www.w3.org/TR/MathML2/> 


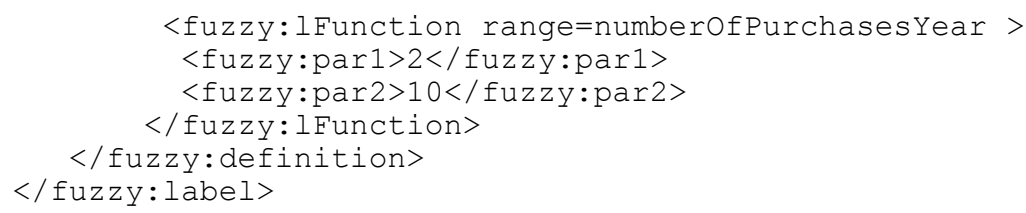

Note that the domain of the fuzzy set is the user entity. Information about the schema (attributes and associations) of UM and DM models are defined in the persistence mechanism, so we only need to specify the entity.

Label sets can be defined in a similar way with a $<$ fuzzy : labelset $>$ tag that includes several label definition, all of them on the same attribute or association.

We have used the Fuzzy Java Toolkit ${ }^{2}$ for the implementation of rules (inference is performed in two phases, one for user modeling and the second for adaptation), mixed with our SHADOWS framework for handling fuzzy types in relational databases. Independent terms are stored as fuzzy relations, which are implemented in a straightforward manner in a relational database by adding attributes to the users table or by including an additional table for each term (both representations are allowed in our implementation). Linguistic label sets are implemented as metadata and values are stored as pairs of keys (label_set, label). In what follows some examples of rules are given; we have specified adaptation rules in a XML syntax that we have tried to keep close to the ongoing research effort of RuleML ${ }^{3}$ [8] (although that effort considers fuzzy logic as one of its future target semantics, it does not support currently fuzziness).

Preferences in Information Browsing Contexts

$A d-h o c$ fuzzy quantified expressions have been used to personalize presentation preferences (number, text size and reading complexity of recommendations) in browsing environments with potentially large information spaces [14]. In this case, the user provides indirect feedback about his/her preferences by rating the items he visited so that evidence can be found over time with common-sense rules in the form "if most long news are rated poorly [by the user] then tend to select shorter ones". Adaptive technologies implemented this way can act as a second filter for a basic information retrieval function. The rule antecedent can be codified as follows:

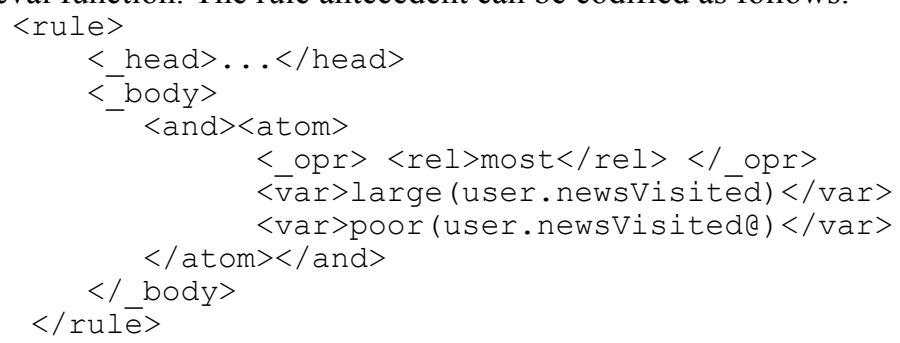

Where 'most' refers to the previously defined quantifier, 'long' is a single label defined on each news' text length and 'poor' is one of the labels in a fuzzy label set 'ratings' (note that they're extensions to RuleML syntax). As news ratings are link

\footnotetext{
$2<$ http://www.iit.nrc.ca/IR_public/fuzzy/fuzzyJToolkit.html>

$3<$ http: //www.dfki.uni-kl.de/ruleml>
} 
attributes of the association between User and News classes, the 'at sign' is used according to the ONN notation described in [1].

Examples on Adaptive Navigation Support

Adaptive hypermedia technologies can be performed with quantified rules as a technique when exploiting quantitative information about user's browsing history or habits. For example, a simple direct guidance [2] behavior can be implemented for the homepage of a university research group that has several sections (e.g. teaching, research, people and resources) with a rule in the form 'if most user sessions browse teaching nodes then $<<$ directly guide the user to that section $>>$ ':

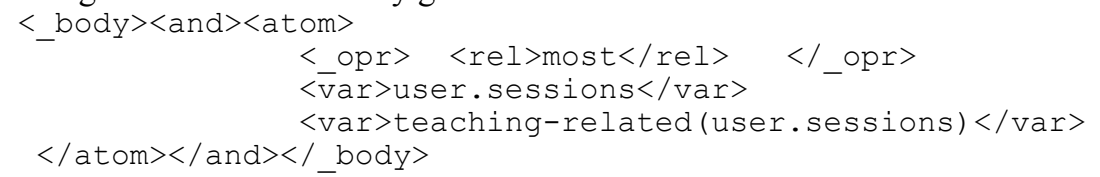

As a second example, ad aptive annotation techniques can augment a link with some kind of information about the node behind the link. A rule like the following can activate a longer description or visual differentiation for links that are not frequently visited or are shortly visited by the user:

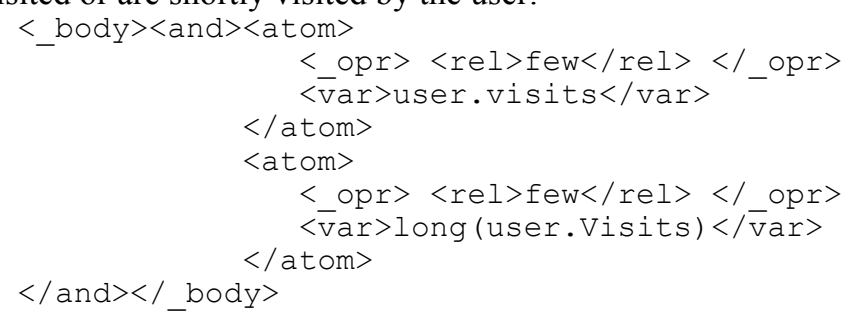

Note that in this latter case, the first quantification is operating on a crisp set, but both atoms yield a fuzzy membership grade due to the application of the quantifier.

\section{Adaptation based on User's Knowledge}

Quantifiers can help in specifying vague or compensatory criteria in adaptive text presentation, for example, in the case of adaptive educational systems, a specific hypermedia node $n$ explaining knowledge item $k$ is showed to user $u$ only if a set of knowledge prerequisites are fulfilled. Then, we have a (fuzzy) relationship between users and knowledge items (i.e. a user 'knows' an item with a specified grade), and another (possibly fuzzy) relation between knowledge items. If we define prerequisites as a term defining the fuzzy subset of the knowledge items that are $k$ 's prerequisites and currentKnowledge as the fuzzy subset of items user u knows, we can relax the presentation criteria with expressions in the form 'if most prerequisites are currentKnowledge then $<<$ show the item $>>$ '. The rule antecedent can be expressed as follows:

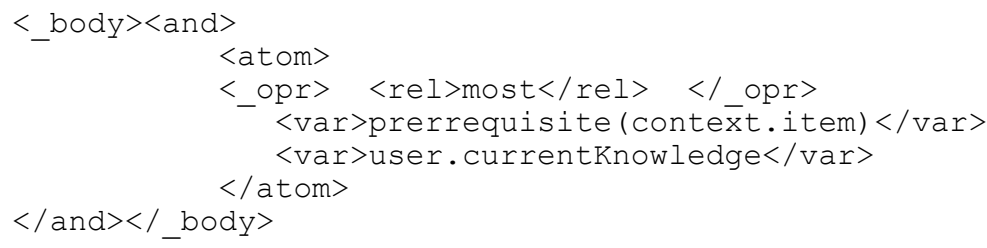


Note that the context is used to refer to the item that is connected to the node that the system is deciding to show or not. The fuzziness of the association currentknowledge implicitly defines a fuzzy subset, and therefore this rule has the semantics of the last example in Table 1.

\section{Conclusions and Future Work}

Quantified expressions enhance the expressive power of rule languages due to its closeness to human language expressions. In the formulation of adaptation rules in AHS, quantified expressions can be used as adaptation methods to specify a number of adaptation technologies. Finally, fuzzy quantification mechanisms can be used to implement adaptation techniques based on quantification, operating on a fuzzy knowledge representation that includes linguistic label sets and fuzzy relations.

Current markup interchange languages for rules need to be complemented to be able to express adaptation rules that operate on complex user and/or domain models. We have defined our own semiformal language combining RuleML, ONN and extensions to the first, and currently a formal definition is in progress.

Future research will address the formulation of complex quantified expressions, applied to general nominal phrases that can involve implicit queries in the user model. In addition, the linguistic adequacy of quantifier implementations should be experimentally validated to ensure they have an appropriate behavior in diverse adaptation contexts, using usability testing techniques (as is common in the field [10]).

\section{Acknowledgements}

This work is supported by "Dirección General de Investigación del Ministerio de Ciencia y Tecnología”, project number TIC2000-0402.

\section{References}

1. Blaha, M. and Premerlani, W.: Object-Oriented Modeling and Design for Database Applications. Prentice Hall, Upper Saddle River, New Jersey (1998)

2. Brusilovsky, P.: Methods and techniques of adaptive hypermedia. User Modeling and User- Adapted Interaction, 6(2/3), Kluwer Academic Publ., The Netherlands (1996) 87129

3. Brusilovsky, P.: Adaptive Hypermedia. User Modeling and User-Adapted Interaction 11(1/2), Kluwer Academic Publ., The Netherlands (2001) 87-110

4. Díaz, P., Aedo, I., Montero, S.: Ariadne, a Development Method for Hypermedia. In: Proceedings of the $12^{\text {th }}$ International Conference on Database and Expert Systems Applications (DEXA'01) (2001) 764-774

5. Dodero, J. M., Sicilia, M.A., García, E.: A Fuzzy Aggregation-Based Reputation Model for e-Learning Exploitation of Public Domain Resources. In: Proceedings of the $4^{\text {th }}$ Interna- 
tional ICSC Symposium on Soft Computing and Intelligent Systems for Industry (SOCO/ISFI'2001), Paisley, Scotland, UK (2001)

6. Fink, J. and Kobsa, A.: A Review and Analysis of Commercial User Modeling Servers for Personalization on the World Wide Web. User Modeling and User-Adapted Interaction 10(3/4), Kluwer Academic Publ., The Netherlands (2000) 209-249

7. Glöckner, I. and Knoll, A.: A Framework for Evaluating Fusion Operators Based on the Theory of Generalized Quantifiers. In: Proceedings of the 1999 IEEE International Conference on Multisensor Fusion and Integration for Intelligent Systems (MFI '99), Taipei, Taiwan (1999)

8. Grosof, B.N.: Standarizing XML Rules. In: Proceedings of the Workshop on E-Business \& the Intelligent Web (IJCAI 2001), Seattle, USA (2001)

9. Herrera, F., Herrera-Viedma, E., Verdegay, J. L.: Aggregating Linguistic Preferences: Properties of LOWA Operator. In: Procceedings of the $5^{\text {th }}$ IFSA World Congress 1995, Sao Paulo, Brasil (1995) 153-156

10. Höök, K.: Evaluating the Utility and Usability of an Adaptive Hypermedia System. Journal of Knowledge-Based Systems 10(5) (1998)

11. Jameson, A.: Numerical Uncertainty Management in User and Student Modeling: An Overview of Systems and Issues. User Modeling and User-Adapted Interaction, 5, Kluwer Academic Publ., The Netherlands (1996) 193-251

12. Kacprzyk, J., Zadrozny S., Yager R.: A fuzzy logic based approach to linguistic summaries of databases. Intl. Journal of Applied mathematics and Computer Science 10(4) (2000)

13. Kobsa, A.: Generic User Modeling Systems. User Modeling and User-Adapted Interaction 11(1/2), Kluwer Academic Publ., The Netherlands (2001) 49-63

14. López, L., Sicilia, M.A., Martínez, J.J., y García, E.: Personalization of Web Interface Structural Elements: A Learning-Scenario Case Study. In: Proceedings of the $3^{\text {rd }}$ Mexican International Conference on Computer Science (ENC'01), Aguascalientes, Mexico (2001)

15. Medina, J. M., Pons, O. and Vila, M.A.: GEFRED: A Generalized Model of Fuzzy Relational Databases, Information Sciences, 76(1/2) (1994) 87-109

16. Moxey, L.M. and Sanford, A.J.: Communicating Quantities: A review of psycholinguistic evidence of the control of perspective by quantifying expressions. Applied Cognitive Psychology, 14 (2000) 237-255

17. Presser, G. Personalization of Newsletters Using Multistage Fuzzy Inference. In: Proceedings of the Fuzzy Days Conference, Lecture Notes in Computer Science 2206, SpringerVerlag (2001) 629-636

18. Rasmussen, D., Yager, R.R.: SummarySQL: A Fuzzy Tool For Data Mining. Intelligent Data Analysis, Elsevier (1997)

19. Sicilia, M.A., Dodero, J.M.: User Stereotype Modeling for Approximate Reasoning-Based Adaptive Enterprise Portals. In: Proceedings of the $10^{\text {th }}$ European-Japanese Conference on Information Modeling and Knowledge Bases, Säariselka, Finland (2000) 177-184

20. Smets, P.: Imperfect information: Imprecision-Uncertainty. In: Motro, A., Smets, P. (eds.) Uncertainty Management in Information Systems: From Needs to Solutions, Kluwer Academic Publishers (1997) 225-254

21. Tsoukalas, L.H., Uhrig, R.E.: Fuzzy and Neural Approaches in Engineering. John Wiley \& Sons, New York (1996)

22. Wu, H., De Kort, E., De Bra, P.: Design Issues for General-Purpose Adaptive Hypermedia Systems. In: Proceedings of the ACM Conference on Hypertext and Hypermedia, Aarhus, Denmark (2001) 141-150

23. Yager, R.R.: Connectives and quantifiers in fuzzy sets. Fuzzy Sets and Systems, 40 (1991) 39-75

24. Zadeh, L.A.: A Computational Approach to Fuzzy Quantifiers in Natural Language. Computing and Mathematics with Applications, 9(1) (1983) 149-184 